matic treatment until spontaneous recovery begins; this may normally be expected within three to four weeks. Recovery usually proceeds in two recognizable phases. There is an initial phase of rapid improvement, which seems to correlate with remyelination of affected peripheral nerves, and a very much slower phase in which reinnervation of denervated muscle takes place by nerve regrowth and sprouting. In most cases recovery is complete, but about $5 \%$ of adult cases have some sequelae. ${ }^{3}$ The prognosis is less good in children. ${ }^{4}$

In adult cases $\operatorname{Ravn}^{5}$ concluded that no prognostic hints could be drawn from the initial course of the disease, but a recent report $^{6}$ has attempted to correlate various clinical features with prognosis for recovery in children suffering from this syndrome. Neither implication of respiratory or bulbar musculature nor early corticosteroid treatment showed any correlation with eventual complete recovery. Those patients who still showed weakness three years after the onset of their disease were the cases in whom severe weakness of distal limb musculature had occurred. The most reliable prognostic indicator was the time interval between maximal weakness and onset of recovery. In those cases in whom improvement was delayed more than 16 days beyond the time when maximal weakness was recorded there was a $96 \%$ probability that recovery would be incomplete.

The duration of maximum symptoms can usually be reliably estimated in the Guillain-Barré syndrome, except in those cases where a chronic relapsing course develops. ${ }^{7}$ If, therefore, these findings are confirmed they may provide a useful indicator of prognosis in children, where as many as a quarter of the cases show weakness more than three years after leaving hospital.

${ }^{1}$ Denny-Brown, D. E., New England fournal of Medicine, 1952, 246, 839. 2 Asbury, A. K., Arnason, B. G., and Adams, R. D., Médicine, 1969, 48. 173.

3 Marshall, J., Brain, 1963, 86, 55.

4 Peterman, A. F., et al., Neurology, 1959, 9, 533.

5 Ravn, H., Acta Neurologica Scandinavica, 1967, Suppl. 30, vol. 43.

6 Eberle, E., et al., Fournal of Pediatrics, 1975, 86, No. 3, 356.

7 Thomas, P. K., et al., Brain, 1969, 92, 589.

\section{Hereditary Pancreatitis}

Pancreatitis is an uncommon disease in children and is usually unexplained, though trauma, mumps and other infections, ascariasis, steroid therapy, and congenital malformations of the pancreas and biliary tree are recognizable aetiological factors. Recurrent pancreatitis is probably even less common, but it may occur in cystic fibrosis, hereditary hyperparathyroidism, hereditary hyperlipidaemia of the type I (fat-induced hyperchylomicronaemia) variety, or as an unexplained familial illness. Indeed hereditary pancreatitis has been claimed to be the most common cause of recurrent pancreatitis in childhood. ${ }^{1}$

Sibert has recently reported a family with hereditary pancreatitis, ${ }^{2}$ the largest kindred to be described in Britain, bringing the total of affected families to over 20 with more than 100 established cases of pancreatitis. Clinically hereditary pancreatitis is very similar to chronic relapsing pancreatitis, and patients complain of recurrent bouts of acute abdominal pain with intervening pain-free periods. The pain is typically epigastric, radiates to the back, and may be accompanied by nausea and vomiting. The biochemical features are common to those found in any form of acute pancreatitis. Eventually exocrine and endocrine insufficiency supervenes and the patient may develop steatorrhoea and diabetes mellitus. ${ }^{134}$ Features peculiar to hereditary pancreatitis are the early onset in childhood, equal incidence for males and females, a preference for Caucasians, and the common and early onset of pancreatic calcification. The pancreatic calcifications develop within the pancreatic ducts ${ }^{5}$ and in children must be distinguished from cystic fibrosis. Possibly patients with hereditary pancreatitis may have a predisposition to pancreatic cancer. ${ }^{3}$

Different kindreds have had singular clinical features. The original families described by Gross ${ }^{6}$ had lysine-cystine aminoaciduria, but these families probably had coexisting hereditary pancreatitis and incompletely recessive cystinuria. Gastrointestinal bleeding features prominently in some families, ${ }^{24}$ and a characteristic of the family described by Sibert was the cessation of abdominal pain once the patients reached 30 to 40 years of age.

The disease is inherited as an autosomal dominant with incomplete penetrance, though the basic inherited disorder is unknown. No specific biochemical defect has been identified, and while hypertrophy of the sphincter of $\mathrm{Oddi}^{7}$ and anomalous narrowing of the pancreatic duct ${ }^{8}$ have been postulated the evidence is not convincing.

1 Kattwinker, J., et al., Pediatrics, 1973, 51, 55.

2 Sibert, J. R., Gut, 1975, 16, 81.

${ }^{3}$ Logan, A. Jr., Schlicke, C. P., and Manning, G. B., American fournal of Surgery, 1968, 115, 112.

${ }^{4}$ McElroy, R., and Christiansen, P. A., American fournal of Medicine, $1972,52,228$

5 Davidson, P., et al., Annals of Internal Medicine, 1968, 68, 88

${ }^{6}$ Gross, J. B., Ganibell, E. E., and Ulrich, J. A., American fournal of Medicine, 1962, 33, 358.

7 Robechek, P. J., American fournal of Surgery, 1967, 113, 819.

${ }^{8}$ Gerber, B. C., Archives of Surgery, 1963, 87, 86.

\section{Antibiotic Cover for Dental Extraction}

The protection of susceptible patients from bacterial endocarditis after dental extraction has been a perennial subject of controversy, but informed medical opinion has recently agreed on two precepts. These are that the antibiotic used should be bactericidal, penicillin being the obvious and usual choice, and that its administration should be begun only shortly before the operation, since pretreatment selects a resistant mouth flora.

To ascertain the current usual practice Durack ${ }^{1}$ sent a questionnaire to 117 dentists in Oxfordshire, of whom 71 replied. All but two believed antibiotics to be of proved value for the purpose, and all but five administered them, the great majority using penicillin. Though this was given in six different forms much the most popular was oral penicillin V, $250 \mathrm{mg}$ 6-hourly. For patients allergic to penicillin 33 gave tetracycline, 20 erythromycin, and 9 cephalosporins-dentists find it much more convenient to write a prescription than to stock and administer an injectable antibiotic, though patients who feel perfectly well can probably not be depended on for selfmedication. Timing and duration were very variable; six started treatment three or four days before extraction, 11 two days, and 31 one day before, and the duration of treatment was anywhere between two and five days.

In fact many of these practices are plainly misguided, and it 
may occasion surprise that the choice of treatment should be left to the dentists, who were far from unanimous in their replies to the question "Do you ask about heart murmurs and rheumatic fever when a new patient presents ?" Should it not be the duty of a physician in charge of a patient with a rheumatic heart lesion to supervise all such treatment?

Durack's own strong views are based not so much on clinical experience (which cannot be so extensive as to afford statistical proof of preventive efficacy) as on experimental study. When catheters are fixed in the right atrium and left ventricle of rabbits via the jugular vein and carotid artery small sterile vegetations form on the valves on which these impinge. A suspension of Strep. viridans may then be injected intravenously and antibiotic treatment given. After 24 hours the animals may be killed and quantitative cultures made from the vegetations. In their main series of such experiments Durack and Petersdorf ${ }^{2}$ found that penicillin sterilized the vegetations only when a high initial level was maintained for nine hoursas is done by a large intramuscular dose of procaine penicillin. The best results were given by a combination of penicillin and streptomycin or of ampicillin and streptomycin, and by vancomycin. Tetracycline, erythromycin, cephalexin, clindamycin, rifampicin, and cotrimoxazole were ineffective. Doubtless in view of spurious claims which have been made for tetracycline Southwick and Durack ${ }^{3}$ tried continued treatment of the experimental infection with this antibiotic for seven days; they found that streptococci still survived in the vegetations. Hence the final recommendation made in Durack's paper on the practice among dentists is that prophylaxis should be either with vancomycin ( $1 \mathrm{~g})$ or with a combination of penicillin 2 mega units, procaine penicillin 500000 units, and streptomycin $1 \mathrm{~g}$; treatment should be given as a single injection shortly before extraction.

Adequate and sufficiently sustained blood levels for a fully dependable effect can be achieved only by parenteral therapy, yet only $12 \%$ of dentists listed this as first choice. The question that nevertheless remains is whether the treatment needs to be as arduous as that proposed. Vancomycin has to be given intravenously, which must detract from its popularity. And is the large dose of penicillin combined with streptomycin really necessary? Is it necessary to give streptomycin at all, unless in a patient already on penicillin treatment, whose mouth streptococci will then be resistant?

One very peculiar case was reported by Durack and Littler, 4 in which bacterial endocarditis was believed to have developed immediately despite two days' cover with generous doses of penicillin and cloxacillin. As pointed out in a subsequent letter 5 "three days is an incredibly short time for endocarditis to appear after dental extraction," and the presumption is that it already existed before the operation. In the main experimental study already quoted a large dose of procaine penicillin alone was effective. Those who have hitherto relied on a single full dose of fortified procaine penicillin may be content still to do so despite these counsels of perfection. Even though the conclusions drawn from it may seem extreme, Durack's experimental study of the problem is much to be commended, and others may be encouraged to emulate it; a modified technique might either confirm or qualify the conclusions drawn and possibly render the clinician's taks less burdensome.

1 Durack, D. T., British Heart fournal, 1975, 37, 478

2 Durack, D. 1., and Petersdorf, R. G., Fournal of Clinical Investigation, $1973,52,592$.

Southwick, F. S., and Durack, D. T., fournal of Clinical Pathology, 1974, $27,261$.

Durack, D. T., and Littler, W. A., Lancet, 1974, 2, 846.

5 Fleming, H. A., Lancet, 1974, 2, 1078.

\section{Pleural Effusion}

Pleural effusion may occur from transudation or exudation. Pleural transudates are clear watery fluids, low in protein, whereas pleural exudates are yellowish and may contain blood and cells. They often clot spontaneously on aspiration and have a higher protein content $(>3 \mathrm{~g} / \mathrm{l})$.

Very small effusions can be seen only on radiological examination by obliteration of the normal costophrenic angle when the patient is in the erect position. Probably there has to be $500 \mathrm{ml}$ or more of fluid before its presence can be detected reliably on physical examination. By far the most important physical sign is stony dullness on percussion over the fluid, and this is usually associated with absent or reduced tactile vocal fremitus and reduced breath sounds, though above or medial to the effusion bronchial breathing is often heard. Vocal resonance may become aegophonic-a bleating quality -but only in the larger effusions is the mediastinum displaced to the opposite side, and this sign may be absent even in large effusions if there is underlying collapse of the lung due, for instance, to a bronchial neoplasm.

Pleural transudates are frequently bilateral and are due to a hydrodynamic imbalance of the forces maintaining capillary blood pressure. They are usually easily diagnosed; there is generally clear clinical evidence of the primary disorder, such as congestive cardiac failure, nephrotic syndrome, hepatic cirrhosis, or malnutrition. A very rare cause of pleural transudates is Meigs's syndrome, ${ }^{1}$ in which usually bilateral effusions are associated with ascites and an ovarian tumour. The collection of fluid in the serous spaces may clear when the tumour is removed; the mechanism is not understood.

Pleural exudates usually occur as a result of primary disease of the underlying lung or pleura. Sometimes pleural transudates and exudates may coexist, and after a patient with congestive cardiac failure and bilateral pleural effusions has responded to therapy one pleural effusion may persist and be found to be due to unsuspected secondary pleural malignant disease. Neoplastic disease is, indeed, among the common causes of pleural exudates, particularly primary carcinoma of the bronchus, of which an effusion may be the earliest physical sign. Pleural exudates also occur due to pleural mesothelioma or metastatic pleural spread from a primary growth elsewhere in the body. Pleural exudates commonly complicate bacterial pneumonia, especially when it has been treated inadequately with antibiotics. Pleural exudates also occur in pulmonary tuberculosis, after pulmonary infarction, and in connective tissue diseases such as rheumatoid arthritis or systemic lupus erythematosus.

It must always be remembered that pleural effusion, especially on the right side, may arise as a result of disease below the diaphragm, such as subphrenic abscess, hepatic amoebiasis, or pyogenic hepatic abscess, and after acute pancreatitis.

Rarer causes of pleural exudates have been described: pleural effusion may be found with primary lymphoedema and yellow discoloration of the nails, the yellow nail syndrome, ${ }^{2}$ and recently pleural exudates, often blood stained, have been reported in patients on chronic dialysis for chronic renal failure. $^{3}$

The cause of most pleural effusions is easily discerned. After assessment of the clinical features and examination of the chest the sputum should be searched for organisms and malignant cells, and a sample of pleural fluid examined for blood, organisms, and cells. Malignant effusions are usually blood stained and accumulate rapidly after aspiration; 